\title{
Point Form Electrodynamics and the Gupta-Bleuler Formalism
}

\author{
W. H. Klink \\ Department of Physics and Astronomy \\ University of Iowa, Iowa City, Iowa, USA
}

October 30, 2018

\begin{abstract}
The Gupta-Bleuler formalism for photons is generalized by choosing the representation of the little group for massless particles, the two dimensional Euclidian group, to be the four dimensional nonunitary representation obtained by restricting elements of the Lorentz group to the Euclidian group. Though the little group representation is nonunitary, it is shown that the representation of the Poincaré group is unitary. Under Lorentz transformations photon creation and annihilation operators transform as irreducible representations of massless particles, and not as four-vectors. As a consequence the polarization vector, which connects the four-vector potential with creation and annihilation operators, is given in terms of boosts, coset representatives of the Lorentz group with respect to the Euclidian group. Several polarization vectors (boost choices) are worked out, including a front form polarization vector. The different boost choices are shown to be related by the analogue of Melosh rotations, namely Euclidian group transformations?.
\end{abstract}

\section{Introduction}

The goal of this series of papers is to construct a relativistic many-body theory of hadrons using the point form of relativistic quantum mechanics,

${ }^{1}$ PACS, 14.70.B 
in which all interactions are vertex interactions, arising from products of fields evaluated at the space-time point zero. In the first of this series of papers [1] such vertex interactions were constructed for the hadronic part of the mass operator. The second paper showed how to construct one-body current operators for arbitrary spin particles [2]. In order that the electromagnetic interaction also be a vertex interaction, the hadronic currents should be coupled to the four-vector potential operator in such a way that the two contracted operators give a scalar density under Lorentz transformations. Moreover the four-vector potential operator should transform as a four-vector under Lorentz transformations, yet be constructed out of photon creation and annihilation operators that transform as one-photon states under Lorentz transformations.

To construct such a vertex, it will be necessary to generalize the GuptaBleuler formulation [3] for photons. For unlike the usual Gupta-Bleuler formulation, the photon creation and annihilation operators should themselves transform under the appropriate irreducible representation of the Poincaré group, namely the massless representations for which the little group is $E(2)$, the two dimensional Euclidean group. The reason is that in order to have an electromagnetic interaction that is a vertex interaction, the polarization vector should be a boost, connecting the creation and annihilation operators with the four-vector potential operator, as is the case for massive particles with spin (see the previous paper, reference [2], Eq.99).

The problem here is well-known; since the two dimensional Euclidean group is noncompact, it has only one dimensional or infinite dimensional unitary representations. The one dimensional representations are usually thought of as providing the relevant representations for a massless spin one particle like the photon and the two polarization states of the photon arise as a consequence of parity. Then it is simple to construct photon creation and annihilation operators with two helicities that transform in the same way as one-photon states. However a problem arises when one wishes to construct a four-vector potential field from these photon creation and annihilation operators, for the "four-vector" potential will not transform as a four-vector under Lorentz transformations [4].

The solution to this problem is also well-known, and goes under the heading of the Gupta-Bleuler formalism [3]; one introduces photon creation and annihilation operators with four components, and eliminates the two spurious components using gauge invariance. However, if the four components transform as a four-vector under Lorentz transformations, there is no natural way 
to link the polarization vector to boosts, as is done in constructing fields for massive particles. This paper will show how photon creation and annihilation operators transforming under $E(2)$ representations naturally link to the four-vector potential field in such a way that the polarization vector is given as a boost, coming from the nonunitary four dimensional representation of the Lorentz group.

One of the advantages of such a procedure is that any boost (coset representative of the Lorentz group with respect to $\mathrm{E}(2))$ can be used as a polarization vector. As will be shown, the usual choice of polarization vector corresponds to a helicity boost. But other choices, such as a front form boost discussed in the appendix, can also be used. In section 2, motivated by induced representation theory, the relevant one-photon states and wave functions are obtained, and the analogue of Wigner rotations for massless particles is derived. Though the four dimensional representation of the Euclidian group is nonunitary, it will be shown that the full Poincaré group representation is unitary. A many-photon theory is generated by photon creation and annihilation operators which have the same Poincaré transformation properties as the single particle photon states. In section 3 the four-vector potential operator is defined in terms of the photon creation and annihilation operators, the link being the polarization vector, that is, a boost Lorentz transformation. What is important here is that the four-vector potential transform as a four-vector under Lorentz transformations, so that, when contracted with the current operators of the previous paper, the electromagnetic vertex be a Lorentz scalar. The section closes with a discussion of gauge transformations for photon creation and annihilation operators and free field four-vector operators.

\section{Photons and the Gupta-Bleuler Formalism}

As is well known the little group for all massless particles is the Euclidian group in two dimensions. A simple proof using $S L(2, C)$ is given in the appendix. If $\Lambda$ is an element of $S O(1,3)$, the proper Lorentz group, then the two dimensional Euclidian group, E(2), can be defined as the subgroup of the proper Lorentz group leaving a standard four vector invariant:

$$
E(2)=\left\{\Lambda \in S O(1,3) \mid \Lambda k^{s t}=k^{s t}\right\},
$$

where $k^{s t}:=(1,0,0,1)$ is the standard four vector. 
To get a Poincaré group representation for massless particles, it is necessary to choose a representation for the little group. Wigner [5] (and others, for example Weinberg, reference [4], page 71) choose the degenerate one dimensional unitary representation for $E(2)$ in which the $E(2)$ translations are trivial, and the rotation angle $\phi$ is represented by $e^{i \lambda \phi}$, with $\lambda$ equal to plus or minus one. Parity connects the plus or minus one helicities, so that photon states can be written as $\mid k, \lambda= \pm 1>$, corresponding to plus or minus helicity states. The problems with this construction have to do with gauge invariance and the link to the four-vector potential operator, which has four components which do not transform among themselves under Lorentz transformations (see reference [4], page 250).

To get around these problems, in this paper another representation for the little group $E(2)$ will be chosen, namely the representation given by the group itself as defined in Eq.1. In terms of the $S L(2, C)$ definition of the two dimensional Euclidian group given in the appendix, Eq.1 can be thought of as a four dimensional nonunitary representation of $E(2)$, for which the representation of an $e_{2}$ element is written as $\Lambda\left(e_{2}\right)$, to indicate the Lorentz transformation representing the Euclidean group element $e_{2}$. The elements of the Euclidian group in this representation can be written explicitly as

$$
\begin{aligned}
\Lambda(\phi)= & {\left[\begin{array}{cccc}
1 & 0 & 0 & 0 \\
0 & \cos \phi & -\sin \phi & 0 \\
0 & \sin \phi & \cos \phi & 0 \\
0 & 0 & 0 & 1
\end{array}\right] } \\
\Lambda(\vec{a})= & {\left[\begin{array}{cccc}
1+|\vec{a}|^{2} / 2 & a_{x} & a_{y} & -|\vec{a}|^{2} / 2 \\
a_{x} & 1 & 0 & -a_{x} \\
-a_{y} & 0 & 1 & a_{y} \\
|\vec{a}|^{2} / 2 & a_{x} & -a_{y} & 1-|\vec{a}|^{2} / 2
\end{array}\right], }
\end{aligned}
$$

where $\vec{a}$ gives the two translations of the Euclidean group.

Any Lorentz transformation can be written as a boost times a Euclidian group element, where the boost $B(k)$ is a Lorentz transformation, that is a coset representative of $S O(1,3)$ with respect to $E(2)$. Boosts have the property of sending $k^{s t}$ to the four vector $k: k=B(k) k^{s t}$, from which it follows that $k \cdot k:=k^{T} g k=k^{\alpha} k_{\alpha}=0 . g$ is the Lorentz metric matrix, $g:=\operatorname{diag}(1,-1,-1,-1)$. The usual boost choice for massless particles is the helicity boost, $B_{H}(k)$, which, as will be shown in section 3 , gives the usual 
polarization vector:

$$
\begin{aligned}
B_{H}(k) & =R(\hat{k}) \Lambda_{z}(|\vec{k}|) \\
& =\left[\begin{array}{cccc}
\operatorname{ch\chi } & 0 & 0 & \operatorname{sh\chi } \\
\operatorname{sh\chi } \hat{k} & \hat{k}_{1} & \hat{k}_{2} & \operatorname{ch\chi } \hat{k}
\end{array}\right]
\end{aligned}
$$

where $R(\hat{k})$ is the rotation matrix taking $\hat{z}$ to the unit vector $\hat{k}, \Lambda_{z}(|\vec{k}|)$ is a Lorentz transformation about the $z$ axis with $|\vec{k}|=e^{\chi}$ and $\hat{k}_{1}=(\cos \phi \cos \theta, \sin \phi \cos \theta,-\sin \theta), \hat{k}_{2}=(-\sin \phi, \cos \phi, 0)$. Another boost choice, a front form boost, is given in the appendix, Eq.42.

Since any Lorentz transformation can be written as $\Lambda=B(k) \Lambda\left(e_{2}\right)$, it follows that the product of two Lorentz transformations, namely $\Lambda B(k)$ can again be decomposed into such a product, namely $B\left(k^{\prime}\right) \Lambda\left(e_{W}\right)$ :

$$
\begin{aligned}
& \Lambda B(k)=B(\Lambda k) \Lambda\left(e_{W}\right) \\
& \Lambda\left(e_{W}\right)=B^{-1}(\Lambda k) \Lambda B(k),
\end{aligned}
$$

where $k^{\prime}=\Lambda k$ is found by applying Eq.6 to $k^{s t} . \Lambda\left(e_{W}\right)$ is the massless analogue of a Wigner rotation(defined for representations of the Poincaré group when particles have nonzero mass). For a given boost, such as a helicity boost defined in Eq.4, the massless Wigner transformation is defined in Eq.7.

With these tools it is possible to define photon states with four degrees of polarization and investigate their transformation properties under Lorentz and space-time transformations:

$$
\begin{aligned}
U_{e_{2}} \mid k^{s t} \alpha> & =\sum \mid k^{s t} \alpha^{\prime}>\Lambda_{\alpha^{\prime} \alpha}\left(e_{2}\right) \\
\mid k, \alpha>: & =U_{B(k)} \mid k^{s t}, \alpha> \\
U_{\Lambda} \mid k, \alpha> & =U_{\Lambda} U_{B(k)} \mid k^{s t}, \alpha> \\
& =\sum \mid \Lambda k, \alpha^{\prime}>\Lambda_{\alpha^{\prime} \alpha}\left(e_{W}\right), \\
U_{a} \mid k, \alpha> & =e^{i k \cdot a} \mid k, \alpha>
\end{aligned}
$$

where $\Lambda\left(e_{W}\right)$ is the Wigner transformation defined in Eq.7; in particular if the Lorentz transformation in Eq.10 is a rotation and the boost a helicity boost, Eq.4, then $\Lambda\left(e_{W}\right)$ becomes a diagonal matrix of phases, exactly as is the case for massive particles with helicity boosts [6].

The transformation properties of photon wave functions are inherited from those of the states. If a state $\mid \phi>$ is written in terms of wave functions 
and basis states,

$$
\left|\phi>=\sum \int \frac{d^{3} k}{k_{0}} \phi(k, \alpha)\right| k, \alpha>
$$

and the action of the Lorentz transformation in Eq.10 on states is transferred to the wave function, one obtains

$$
\left(U_{\Lambda} \phi\right)(k, \alpha)=\sum \Lambda_{\alpha, \alpha^{\prime}}\left(e_{W}\left(k, \Lambda^{-1}\right)\right) \phi\left(\Lambda^{-1} k, \alpha^{\prime}\right) .
$$

Here $d^{3} k / k_{0}$ is the Lorentz invariant measure with $k_{0}=|\vec{k}|$. To be more mathematically precise, the Hilbert space discussed in the following paragraphs, and the transformation properties of the Hilbert space elements under Lorentz transformations given in Eq.13 could all be derived directly from induced representation theory []]. The only somewhat unusual feature would be that the representation of the Euclidian group is four dimensional, rather than the more usual one dimensional representation. Such a mathematical background would be necessary to prove the irreducibility of the massless representation, a subject that will not be pursued further in this paper.

To show that the representation defined by Eq.13 is unitary, it is necessary to define an inner product on photon wave functions. Since the representation of the little group, Eqs.2,3, is not unitary, the usual Hilbert space of square integrable functions will not lead to a unitary representation for the Poincaré group. But the representation of the little group is in terms of Lorentz matrices which satisfy $\Lambda g \Lambda^{T}=g$; this property can be used to define an inner product which produces a unitary representation for the Poincaré group. Define a photon inner product as

$$
(\phi, \psi):=-\sum \int \frac{d^{3} k}{k_{0}} \phi^{*}(k, \alpha) g_{\alpha, \alpha} \psi(k, \alpha)
$$

then the representation defined by eq.13 is unitary, that is $\left\|U_{\Lambda} \phi\right\|^{2}=\left\|U_{a} \phi\right\|^{2}=$ $\|\phi\|^{2}$.

But the inner product as defined in Eq.14 is not positive definite (for example choose $\phi(k, 0)=\phi(k)$, with the other components zero). As is well known [3], to get around this problem, the zero component of the wave function is chosen to equal the third component of the wave function:

$$
\begin{aligned}
\phi(k, 0) & =\phi(k, 3), \\
\sum k_{\alpha}^{s t} g_{\alpha, \alpha} \phi(k, \alpha) & =0 .
\end{aligned}
$$


Using Eq.13 it is easy to see that the condition, Eq.16, is Lorentz invariant. This allows one to define the photon Hilbert space $H_{\gamma}$ as

$$
H_{\gamma}=\{\phi(k, \alpha) \mid \phi(k, 0)=\phi(k, 3)\} \text {, }
$$

with the inner product given in Eq.14, resulting in a positive definite inner product, in which the zero and three components of the wave function do not contribute to the inner product because of Eq.15. Moreover, quantities such as the expectation value of the energy, $\left(\phi, P_{0} \phi\right)$ are also seen to be positive definite, with the value zero occurring only when the one and two component parts of the wave function are zero.

With this background it is now possible to introduce many photon states and wave functions, living in the Fock space generated by sums of symmetrized tensor products of $H_{\gamma}$, through photon creation and annihilation operators, whose transformation properties are inherited from the one particle photon properties:

$$
\begin{aligned}
\mid k, \alpha> & =c^{\dagger}(k, \alpha) \mid 0> \\
c(k, \alpha) \mid 0> & =0, \alpha=0,1,2,3 \\
{\left[c(k, \alpha), c^{\dagger}\left(k^{\prime}, \alpha^{\prime}\right)\right] } & =-g_{\alpha, \alpha^{\prime}} k_{0} \delta^{3}\left(\vec{k}-\vec{k}^{\prime}\right) \\
U_{\Lambda} c(k, \alpha) U_{\Lambda}^{-1} & =\sum c\left(\Lambda k, \alpha^{\prime}\right) \Lambda_{\alpha, \alpha^{\prime}}\left(e_{W}\right) \\
U_{a} c(k, \alpha) U_{a}^{-1} & =e^{-i k \cdot a} c(k, \alpha) \\
P_{\mu}^{\gamma} & =-\sum \int \frac{d^{3} k}{k_{0}} k_{\mu} c^{\dagger}(k, \alpha) g_{\alpha, \alpha} c(k, \alpha) .
\end{aligned}
$$

The important difference between the usual Gupta-Bleuler analysis and this paper is seen in Eq.21. The creation and annihilation operators do not transform as four-vectors under Lorentz transformations, but as irreducible representations of the Poincaré group for massless particles, in which the little group representation is a four dimensional nonunitary representation of the two dimensional Euclidean group.

The wave function condition,Eq.15, now becomes the annihilation operator condition

$$
\sum k_{\alpha}^{s t} c(k, \alpha) \mid \phi>=0
$$

for all $k$ and for all $\mid \phi>$ in the Fock space. By applying a Lorentz transformation to Eq.24 and using Eq.21, it is straightforward to show that Eq.24 
is a Lorentz invariant condition. Moreover, it guarantees that no timelike or longitudinal components will contribute to the inner product. That is, if $\left|\phi_{n}\right\rangle$ is an n-photon state, Eq.24 guarantees that the $\alpha_{i}=0$ components will cancel the $\alpha_{i}=3$ components in the n-photon wavefunction. Hence the inner product,

$$
\begin{aligned}
<\phi_{n} \mid \phi_{n}> & =(-1)^{n} \sum_{\alpha_{i}} \int \prod_{i} \frac{d^{3} k_{i}}{k_{0 i}}\left|\phi_{n}\left(k_{1} \alpha_{1} \ldots k_{n} \alpha_{n}\right)\right|^{2} g_{\alpha_{1}, \alpha_{1}} \ldots g_{\alpha_{n}, \alpha_{n}} \\
& =\sum_{\alpha_{i}=1,2} \int \prod_{i} \frac{d^{3} k_{i}}{k_{0 i}}\left|\phi_{n}\left(k_{1} \alpha_{1} \ldots k_{n} \alpha_{n}\right)\right|^{2},
\end{aligned}
$$

where all the $\alpha_{i}=0$ components have cancelled with the $\alpha_{i}=3$ components, so that the norm is always nonnegative.

\section{The Free Four-Vector Potential Operator}

In this section, as in all the papers in this series, fields will be defined as translates of the four-momentum operator from the space-time point zero. In this paper the four-momentum operator is taken to be the photon fourmomentum operator, defined in Eq.23, while in the next paper [8], it will include matter and electromagnetic four-momentum operators. However, the four-vector potential operator at the space-time point zero is always defined by

$$
A_{\mu}(0):=-\sum \int \frac{d^{3} k}{k_{0}} B_{\mu \alpha}(k) g_{\alpha, \alpha}\left(c(k, \alpha)+c^{\dagger}(k, \alpha)\right) .
$$

As a consequence of this definition and the transformation properties of the photon creation and annihilation operators, Eq.21, the four-vector potential operator will transform as a four-vector under Lorentz transformations, $U_{\Lambda} A_{\mu}(0) U_{\Lambda}^{-1}=\left(\Lambda^{-1}\right)_{\mu}^{\nu} A_{\nu}(0)$. Further the polarization vector, usually written as $\epsilon_{\mu \alpha}(k)$ (see for example, Schweber, reference [9], page 249), is seen to be the boost matrix discussed after Eq.3. Usually a helicity boost is chosen (see Eq.4) but it is clear that any other boost will serve equally well, for example the boost defined in the appendix, Eq.42.

The free four-vector potential operator at the space-time point $x$ is defined to be

$$
A_{\mu}(x):=e^{i P^{\gamma} \cdot x} A_{\mu}(0) e^{-i P^{\gamma} \cdot x}
$$




$$
=-\sum \int \frac{d^{3} k}{k_{0}} B_{\mu \alpha}(k) g_{\alpha, \alpha}\left(e^{-i k \cdot x} c(k, \alpha)+e^{i k \cdot x} c^{\dagger}(k, \alpha)\right)
$$

From this definition it follows that this operator is local, that is, the commutator $\left[A_{\mu}(x), A_{\nu}(y)\right]$ is zero for $(x-y)^{2}$ spacelike. Eq.28 can also be used to relate the generalized Gupta-Bleuler formalism developed in this paper with the usual Gupta-Bleuler formalism. If the boost in Eq.28 is chosen to be a helicity boost, Eq.5, then the annihilation operator transforming as a fourvector under Lorentz transformations is related to the annihilation operator transforming as a one particle state under Lorentz transformations (Eq.21) by $c(k, \mu)=B_{\mu \alpha}(k) g_{\alpha, \alpha} c(k, \alpha)$.

Finally, the positive frequency part of the four vector field satisfies a (nonlocal) Lorentz gauge condition:

$$
\begin{aligned}
A_{\mu}^{+}(x): & =-\sum \int \frac{d^{3} k}{k_{0}} B_{\mu \alpha}(k) g_{\alpha, \alpha} e^{-i k \cdot x} c(k, \alpha) \\
\partial A_{\mu}^{+}(x) / \partial x_{\mu} & =i \sum \int \frac{d^{3} k}{k_{0}} k^{\mu} B_{\mu \alpha}(k) g_{\alpha, \alpha} e^{-i k \cdot x} c(k, \alpha) \\
& =i \sum \int \frac{d^{3} k}{k_{0}} e^{-i k \cdot x} k_{\alpha}^{s t} c(k, \alpha) \\
& =0
\end{aligned}
$$

where use has been made of the fact that an inverse boost on the four vector $k$ results in the four vector $k^{s t}$.

This section concludes with a discussion of gauge invariance for the creation and annihilation operators and the four-vector potentials. If $c(k, \alpha)$ is the annihilation operator defined in Eq.19 ff, then the gauge transformed annihilation operator is defined to be

$$
c^{\prime}(k, \alpha):=c(k, \alpha)+k_{\alpha}^{s t} f(k) I,
$$

where $f(k)$ is a complex function of the four vector $k$ and $I$ is the identity operator ${ }^{2}$. $c^{\prime}(k, \alpha)$ must satisfy the same conditions as $c(k, \alpha)$, namely Lorentz covariance (Eq.21), boson commutation relations (Eq.20), and the subsidiary condition (Eq. 24). The subsidiary condition for $c^{\prime}(k, \alpha)$ follows immediately

\footnotetext{
${ }^{2}$ It would also be possible to make $f(k) I$ an operator, say $d(k)$, which acts on the full photon Fock space. This is equivalent to introducing an auxiliary massless scalar field. Requiring that $c^{\prime}(k, \alpha)$ satisfy the same properties as $c(k, \alpha)$ then puts requirements on $d(k)$ see reference [10] and in particular page 75 .
} 
from the definition, since $k^{s t} \cdot k^{s t}=0$. Also the commutation relations follow since the term added to $c(k, \alpha)$ is a multiple of the identity operator.

The Lorentz condition can be written as

$$
\begin{aligned}
U_{\Lambda} c^{\prime}(k, \alpha) U_{\Lambda}^{-1} & =\sum c\left(\Lambda k, \alpha^{\prime}\right) \Lambda_{\alpha^{\prime} \alpha}\left(e_{W}\right)+k_{\alpha}^{s t} f(k) I \\
& =\sum\left(c\left(\Lambda k, \alpha^{\prime}\right)+k_{\alpha^{\prime}}^{s t} f(k) I\right) \Lambda_{\alpha^{\prime} \alpha}\left(e_{W}\right) \\
& =\sum c^{\prime}\left(\Lambda k, \alpha^{\prime}\right) \Lambda_{\alpha^{\prime} \alpha}\left(e_{W}\right),
\end{aligned}
$$

the desired result if $f(k)=f(\Lambda k)$, that is, $f(k)$ is a Lorentz scalar. Gauge transformations have the effect of adding or subtracting equal amounts of timelike and longitudinal components and thus do not change the norm of many-photon wave functions.

Finally, the positive frequency part of the gauge transformed four vector field is also conserved:

$$
\begin{aligned}
A_{\mu}^{\prime}(x): & =\sum \int \frac{d^{3} k}{k_{0}} B_{\mu \alpha}(k) e^{-i k \cdot x} g_{\alpha, \alpha} c^{\prime}(k, \alpha) \\
& =A_{\mu}^{+}(x)+\sum \int \frac{d^{3} k}{k_{0}} k_{\mu} e^{-i k \cdot x} f(k) I ; \\
\partial A_{\mu}^{+}(x) / \partial x_{\mu} & =\partial A_{\mu}^{+}(x) / \partial x_{\mu}-i \sum \int \frac{d^{3} k}{k_{0}} k \cdot k e^{-i k \cdot x} f(k) I \\
& =0 .
\end{aligned}
$$

\section{Conclusion}

In order to be able to write electromagnetic interactions as vertex interactions, it is necessary to generalize the Gupta-Bleuler formalism so that photon creation and annihilation operators transform as single particle states under Lorentz transformations. As first pointed out by Wigner, the little group for massless particles is the two dimensional Euclidian group $\mathrm{E}(2)$; but the choice of representation for $\mathrm{E}(2)$ is not given a priori. Wigner [5] (and later others, including Weinberg, reference [国) choose the degenerate one dimensional representation of $\mathrm{E}(2)$, in which the action of the translations in $\mathrm{E}(2)$ are trivial. While it is possible to obtain the usual photon states and wavefunctions with such a representation of $\mathrm{E}(2)$, troubles arise not only with gauge invariance, but also with providing the natural link between fields and 
photon creation and annihilation operators, of the sort available for massive particles with spin (see for example, reference [4, page 233).

In this paper the massless little group representation is chosen to be the four dimensional nonunitary representation of $\mathrm{E}(2)$, obtained by restricting elements of the Lorentz group to $\mathrm{E}(2)$; the form of these $\mathrm{E}(2)$ elements, as Lorentz transformations, is given in Eqs.2,3. Such a representation must be nonunitary, since it is a finite dimensional representation of a noncompact group. Nevertheless the representation of the full Poincaré group is unitary. Such a result makes use of the fact that E(2) matrices are Lorentz matrices; by suitably modifying the inner product, the resulting Poincaré representation is unitary and the inner product agrees with that given by the Gupta-Bleuler formalism.

The inner product that makes the representation of the Poincaré group unitary is not positive definite. So-as with the Gupta-Bleuler formalism- the photon Hilbert space is defined as the subspace of wavefunctions for which the timelike and longitudinal components are equal. Such a subspace is a Poincaré invariant subspace. Many-photon states and wavefunctions can then be defined in terms of photon creation and annihilation operators. These creation and annihilation operators do not however transform as four vectors, as is usually the case (see reference [9], page 243); rather under Lorentz transformations they transform as Euclidian analogues of Wigner rotations (see Eq.21), which is the natural generalization of the transformation properties for massive particles with spin. Because of these transformation properties, the proof of the operator condition that longitudinal and time-like components cancel is Lorentz invariant is particularly simple.

The main result of this paper concerns the link between the four-vector potential operator and photon creation and annihilation operators. For massive particles with spin this link is always given by Lorentz group representations of boosts, coset representatives of $\mathrm{SO}(1,3)$ with respect to the rotation group. For example the usual spinor functions for spin $1 / 2$ fermions are boosts, usually canonical spin boosts. But there are many boost possibilities, such as helicity or front form boosts [11]. Similarly for massless particles boosts are coset representatives of $\mathrm{SO}(1,3)$ with respect to $\mathrm{E}(2)$, and provide the link between the four-vector potential and photon creation and annihilation operators. That is, polarization vectors are boost representatives, coset choices of $\mathrm{SO}(1,3)$ with respect to $\mathrm{E}(2)$. The usual polarization choice is the helicity boost, given in Eq.5. But just as there are many different boost choices for massive particles, all connected by Melosh rotations [11], [12], so 
too there are many boost choices for massless particles, all connected by the analogue of Melosh rotations, namely E(2) transformations. An example of a non-helicity polarization vector, a front form boost for massless particles in given in the appendix, Eq.42.

Gauge transformed photon creation and annihilation operators with the correct Lorentz (Eq.21) and subsidiary (Eq.24) conditions affect only the time-like and longitudinal polarizations, leaving the transverse parts unchanged. Using the connection between four-vector potentials and creation and annihilation operators, the usual gauge transformations for the positive frequency part of the four-vector potentials are obtained, as well as the fact that under such a gauge transformation the Lorentz gauge condition remains invariant. Since the four-vector potential operator transforms as a four-vector under Lorentz transformations, it can be coupled to the current operators defined in the previous paper, to form the electromagnetic vertex for particles of any spin, which is the starting point for constructing the electromagnetic mass operator.

To conclude it should be pointed out that the procedures applied here to photons can equally well be applied to massless spin two (or for that matter to arbitrary spin) particles, namely gravitons. The construction of the relevant nonunitary representations of the Euclidian group, as well as the construction of different polarization tensors, will be discussed in another paper.

\section{A Appendix: SL $(2, \mathrm{C})$ and Massless Particles}

In section 2 all operations were carried out using the Lorentz group $\mathrm{SO}(1,3)$. In this appendix the fact that $\mathrm{SL}(2, \mathrm{C})$ is the covering group of the Lorentz group is used to derive some results in a more transparent way. Under a Lorentz transformation $\Lambda$, a four vector $k$ goes to $k^{\prime}=\Lambda k$. Such a transformation is carried out in $\mathrm{SL}(2, \mathrm{C})$ by replacing the four-vector $k$ by the hermitian matrix $H(k)$, with

$$
\begin{aligned}
H\left(k^{\prime}\right) & =A H(k) A^{\dagger} \\
H(k) & =\left[\begin{array}{ll}
k_{+} & k_{\perp}^{*} \\
k_{\perp} & k_{-}
\end{array}\right] .
\end{aligned}
$$

$A$ is an element of $\mathrm{SL}(2, \mathrm{C})$ and $k_{ \pm}=k_{0} \pm k_{z}, k_{\perp}=k_{x}+i k_{y}$.

The standard vector $k^{s t}$ introduced in Eq.3 becomes $H\left(k^{s t}\right)$ and the little 
group is the subgroup of $\mathrm{SL}(2, \mathrm{C})$ that leaves $H\left(k^{s t}\right)$ invariant:

$$
\begin{aligned}
E(2) & =\left\{A \in S L(2, C) \mid H\left(k^{s t}\right)=A H\left(k^{s t}\right) A^{\dagger}\right\} \\
& =\left\{e_{2}(\phi, a)\right\} \\
& =\left\{\left[\begin{array}{cc}
e^{i \phi / 2} & a e^{i \phi / 2} \\
0 & e^{-i \phi / 2}
\end{array}\right]\right\},
\end{aligned}
$$

where now $a=a_{x}+i a_{y}$ gives the two translations. Written in this way it is straightforward to show that the elements in Eq.38 combine as E(2) elements.

Any element of $\mathrm{SL}(2, \mathrm{C})$ can be decomposed into boosts (coset representatives) with respect to $\mathrm{E}(2)$. A natural choice is a front form boost,

$$
\begin{aligned}
B_{F}(k): & =\left[\begin{array}{cc}
\sqrt{k_{+} / 2} & 0 \\
k_{\perp} / \sqrt{2 k_{+}} & \sqrt{2 / k_{+}}
\end{array}\right] \\
H(k) & =B_{F}(k) H\left(k^{s t}\right) B_{F}^{\dagger}(k),
\end{aligned}
$$

for then

$$
A=B_{F}(k) e_{2}(\phi, a)
$$

and the parameters of A are readily expressed in terms of $k$ and the Euclidian parameters $\phi$ and $a$.

The correspondence between elements of $\mathrm{SL}(2, \mathrm{C})$ and $\mathrm{SO}(1,3)$ [13] then gives the front form boost, Eq.39, as an $\mathrm{SO}(1,3)$ element, suitable for a polarization vector:

$$
B_{F}(k)=\left[\begin{array}{cccc}
k_{0} / 2+1 / k_{+} & k_{x} / k_{+} & k_{y} / k_{+} & k_{0} / 2-1 / k_{+} \\
k_{x} / 2 & 1 & 0 & k_{x} / 2 \\
k_{y} / 2 & 0 & 1 & k_{y} / 2 \\
k_{z} / 2-1 / k_{+} & -k_{x} / k_{+} & -k_{y} / k_{+} & k_{z} / 2+1 / k_{+}
\end{array}\right] .
$$

Since an arbitrary Lorentz group element can now be decomposed in (at least) two ways, namely $\Lambda=B_{H}(k) e_{2}^{\prime}=B_{F}(k) e_{2}$, it follows that $B_{F}(k)=$ $B_{H}(k) e_{2}^{\prime} e_{2}^{-1}$, that is, all boosts are related by $e_{2}$ elements, which is the massless analogue of Melosh rotations [12].

\section{References}

[1] W. H. Klink, "Constructing Point Form Mass Operators from Vertex Interactions", submitted. 
[2] W. H. Klink, "Local Current Operators for Arbitrary Spin Particles", submitted.

[3] K. Bleuler, Helv.Phys.Acta, 23 (1950) 567, S. N. Gupta, Proc. Phys. Soc. (London), A63 (1950) 681; see also S. S. Schweber, An Introduction to Relativistic Quantum Field Theory (Harper and Row, New York, USA, 1962), chapter 9.

[4] S. Weinberg, The Quantum Theory of Fields, Volume I (Cambridge University Press, Cambridge, England, 1995).

[5] E. P. Wigner, Ann. Math. 40 (1939) 149.

[6] W. H. Klink, Ann. Phys. (N.Y.) 213 (1992) 31.

[7] R. Warren, W. H. Klink, J. Math. Phys. 11 (1970) 1155; G. Mackey, The Theory of Induced Representations (University of Chicago Press, Chicago, USA, 1955).

[8] W. H. Klink, "Point Form Electrodynamics and the Construction of Conserved Current Operators", submitted.

[9] See for example, Schweber, reference [3], page 243.

[10] N. Nakanishi, I. Ojima, Covariant Operator Formalism of Gauge Theories and Quantum Gravity (World Scientific Lecture Notes in Physics, Volume 27, Singapore, 1990).

[11] W. H. Klink, Phys.Rev.C58 (1998) 3617.

[12] H. D. Melosh, Phys.Rev.D9 (1974) 1095; also reference 11, page 3625.

[13] M.A. Naimark, Linear Representations of the Lorentz Group, (Pergamon Press, Oxford, England, 1964), page 122. 